TRANSACTIONS OF THE

AMERICAN MATHEMATICAL SOCIETY

Volume 214, 1975

\title{
WEAKLY SMOOTH CONTINUA
}

BY

\section{LEWIS LUM}

ABSTRACT. We define and investigate a class of continua called weakly smooth. Smooth dendroids, weakly smooth dendroids, generalized trees, and smooth continua are all examples of weakly smooth continua. We generalize characterizations of the above mentioned examples to weakly smooth continua. In particular, we characterize them as compact Hausdorff spaces which admit a quasi order satisfying certain properties.

Introduction. A dendroid $X$ is smooth at the point $p$ if whenever $x_{n}$ is a net in $X$ the condition $\lim _{n} x_{n}=x$ implies $\operatorname{Lim}_{n}\left[p, x_{n}\right]=[p, x]$, where $[p, x]$ denotes the unique subcontinuum of $X$ irreducible between $p$ and $x$. Smooth dendroids were investigated by Charatonik and Eberhart [3]. The nonmetric analog of smooth dendroids, generalized trees, was studied by Ward [20] .

G. R. Gordh, Jr. [8] generalized this notion to smooth continua by imposing the smoothness condition on continua which are hereditarily unicoherent at p. In [5] various characterizations of smooth continua are obtained; smooth metric continua are discussed in [15]; and a quasi order characterization was obtained in [13].

In [14] smooth dendroids were generalized by relaxing the smoothness condition. A dendroid $X$ is weakly smooth at $p$ if whenever $x_{n}$ is a convergent net in $X$ there exists a point $x \in X$ such that $\operatorname{Li}_{n}\left[p, x_{n}\right]=[p, x]$. Analogs of the well known characterizations of smooth dendroids were proved.

The purpose of this paper is to define and investigate weakly smooth continua. We generalize results in [5] and [14] to weakly smooth continua as well as obtain other characterizations. In particular, we characterize weakly smooth continua as compact Hausdorff spaces which admit a quasi order satisfying certain properties.

We use the following notation: the symbol $\square$ denotes the empty set; and if $A \subseteq Y \subseteq X$ then $\operatorname{cl} A\left(\operatorname{cl}_{Y} A\right)$ denotes the closure of $A$ (in $Y$ ) and int $A$ (int $Y$ ) denotes the interior of $A$ (in $Y$ ).

Presented to the Society, January 23, 1975; received by the editors February 20, 1974 AMS (MOS) subject classifications (1970). Primary 54F20; Secondary 54F65, 54F05.

Key words and phrases. Hereditarily unicoherent at a point, indecomposable continuum, irreducible continuum, partial order, quasi order, smooth continuum, type $A^{\prime}$, weak cut point order, weakly smooth continuum. 
1. Preliminaries. A continuum is a nondegenerate compact connected Hausdorff space. A continuum is irreducible if it contains two points with no proper subcontinuum containing them. An arc is a continuum (not necessarily metrizable) with exactly two noncut points. A continuum is decomposable if it can be expressed as the union of two proper subcontinua; otherwise, it is indecomposable.

An irreducible continuum is of type $A^{\prime}$ [7] if each subcontinuum with nonvoid interior is decomposable. We define a continuum to be of type $A^{\prime}$ at the point $p$ if each subcontinuum which is irreducible between $p$ and some other point is of type $A^{\prime}$.

A continuum is hereditarily unicoherent if the intersection of any two subcontinua is connected. It is hereditarily unicoherent at the point $p$ [8] if the intersection of any two subcontinua, each of which contains $p$, is connected.

An arboroid is a hereditarily unicoherent arcwise connected continuum. A tree is a hereditarily unicoherent locally connected continuum. A dendroid (dendrite) is a metrizable arboroid (tree). These definitions are known to be equivalent to the original definitions (see [4], [19], and [20]).

Throughout this paper we use the letter $X$ to denote a continuum which (i) is hereditarily unicoherent at $p$, and (ii) has the property that all of its indecomposable subcontinua are irreducible. Note that arboroids satisfy (i) by definition and (ii) by a result of Bellamy [1].

For each $x \in X$, let $[p, x]$ denote the unique subcontinuum irreducible between $p$ and $x$. The weak cut point order with respect to $p$, denoted $\leqslant_{p}$, is defined by $x \leqslant_{p} y$ if and only if $x \in[p, y]$.

For a subset $A \subseteq X$ let

$L(A)=\left\{x \in X \mid x \leqslant_{p} a\right.$ for some $\left.a \in A\right\}$,

$M(A)=\left\{x \in X \mid a \leqslant_{p} x\right.$ for some $\left.a \in A\right\}$, and

$E(A)=L(A) \cap M(A)$.

Note that for each $x \in X, L(x)=[p, x]$ and $E(x)=\{y \in X \mid[p, x]=[p, y]\}$.

A subset $C \subseteq X$ is a chain (with respect to $\leqslant_{p}$ ) if $C \times C \subseteq \Gamma_{p} \cup \Gamma_{p}^{-1}$ where $\Gamma_{p}=\left\{(x, y) \in X \times X \mid x \leqslant_{p} y\right\}$ and $\Gamma_{p}^{-1}=\left\{(x, y) \in X \times X \mid(y, x) \in \Gamma_{p}\right\}$.

An element $a$ in a subset $A \subseteq X$ is minimal (maximal), whenever $x \leqslant_{p} a$ $\left(a \leqslant_{p} x\right)$ in $A$ implies $a \leqslant_{p} x \quad\left(x \leqslant_{p} a\right)$. The set of all minimal (maximal) elements of $A$ is denoted $\min A(\max A)$. If $z \in A$ and $z \leqslant_{p} a$ for all $a \in A$ then $z$ is called a zero of $A$.

The symbols $x \leqslant_{p} y, x \in[p, y], x \in L(y)$, and $(x, y) \in \Gamma_{p}$ will be used interchangeably. In case $x \leqslant_{p} y$ and $x \notin E(y)$ we will write $x<_{p} y$.

Although $\leqslant_{p}$ may not be antisymmetric, it is always reflexive and transitive. Such a relation is called a quasi order. An antisymmetric quasi order is called a partial order. 
LEMMA 1.1. Let $x \in X-\{p\}$. Then:

(i) $E(p)=p$;

(ii) int $_{L(x)} E(x)=$;;

(iii) $E(x)$ is connected;

(iv) if $X$ is of type $A^{\prime}$ at $p$, then $E(x)$ is closed;

(v) if $A \subseteq X$ is closed, then $\min A \neq \square$;

(vi) each subcontinuum of $X$ has a zero;

(vii) if $\leqslant_{p}$ is a partial order (in particular, if $X$ is arcwise connected), then $X$ is hereditarily unicoherent.

Proof. (i) is clear. (ii) follows from [9, Theorem 3-44, p. 140]. (iii) is a special case of [6, Theorem 1.2]. For (iv), the proof of [17, Theorem 7, p. 13] generalizes to nonmetric continua. See [21, Theorem 1, p. 146] for the proof of (v). To prove (vi) let $Y$ be a subcontinuum of $X$ and let $z \in \min Y$. Then $[p, z]$ $\cap Y \subseteq E(z)$ and $[p, z] \cup Y$ is a continuum which is hereditarily unicoherent at $p$. Hence for each $y \in Y,[p, y] \subseteq[p, z] \cup Y$. Consequently, $\square \neq[p, y] \cap([p, z]$ $\cap Y) \subseteq E(z)$. It follows that $z \leqslant p$; that is, $z$ is a zero of $Y$. Finally, the proof of (vii) is similar to the proof of [5, Theorem 2.2, p. 63].

Our first theorem is a partial generalization of $[7$, Theorem 3.7, p. 653].

THEOREM 1.2. If $X$ is a chain then $X$ is irreducible between $p$ and some other point.

Proof. Index $X=\left\{x_{n}\right\}$ so that $x_{m} \leqslant x_{n}$ whenever $m \leqslant n$. Then $X=$ $\bigcup_{n}\left[p, x_{n}\right]$ is the union of an increasing nest of irreducible continua.

Note that for each $x \in X$, there exists $y \in X$ such that $x<_{p} y$. (If this were not the case we would be done.)

Claim 1. Suppose $Y$ is a subcontinuum of $X$ containing $p$. If $Y$ contains $x_{n}$ for arbitrarily large $n$ then $Y=X$.

Proof of claim. For any $x_{m} \in X$ choose $x_{n} \in Y$ such that $x_{m} \leqslant x_{p}$. Since $Y$ is hereditarily unicoherent at $p, x_{m} \in\left[p, x_{n}\right] \subseteq Y$. It follows that $Y=X$.

Claim 2. For each $x \in X$, the set $\mathrm{cl} M(x)$ is connected.

Proof of claim. We first show $N=X-[p, x]$ is connected. Let $A$ and $B$ be separated sets whose union is $N$. Since $x_{n} \in N$ for all $x<_{p} x_{n}$, we may assume $x_{n} \in A$ for arbitrarily large $n$. From Claim 1 we infer $X=[p, x] \cup \operatorname{cl} A$ and hence $B=\square$. Thus $N$ is connected.

Now $M(x)=N \cup E(x)$ is the union of two connected sets. Since $\mathrm{cl} M(x)=$ $\operatorname{cl} N \cup \operatorname{cl} E(x)$ it suffices to show $\operatorname{cl} N \cap \operatorname{cl} E(x) \neq \square$. Suppose $\operatorname{cl} N \cap \operatorname{cl} E(x)=$ $\square$; then in particular, $x \notin \mathrm{cl} N$. Choose any $z \in \mathrm{cl} N \cap[p, x]$ and note that $x \notin$ $[p, z]$. But according to Claim $1,[p, z] \cup \operatorname{cl} N=X$. This contradiction proves the claim. 
Claim 3. Let $z$ be a cluster point of the net $X=\left\{x_{n}\right\}$. If $z<_{p} x$ then $x$ is also a cluster point of the net $x_{n}$. In particular, there exists $z \in X$ such that whenever $z \leqslant_{p} x, x$ is a cluster point of the net $x_{n}$.

Proof of claim. Suppose $z<_{p} x$ and $x$ is not a cluster point of the net $x_{n}$. Then there exists $y \in X$ and an open neighborhood $U$ in $X$ satisfying $x \in U \subseteq$ $[p, y]-E(y)$. By the selection of $z$ it follows that $z \in \mathrm{cl} M(y)$. Hence by Claim $1, X=[p, z] \cup \mathrm{cl} M(y)$. This is a contradiction since $x \notin[p, z]$ and $\operatorname{cl} M(y) \subseteq \operatorname{cl}(X-U)$ implies $x \notin \operatorname{cl} M(y)$.

For the remainder of the proof assume $z$ is a point in $X$ such that $x$ is a cluster point of the net $x_{n}$ whenever $z \leqslant_{p} x$.

Claim 4. The continuum $\mathrm{cl} M(z)$ is indecomposable.

Proof of claim. Let $H \subseteq \mathrm{cl} M(z)$ be a subcontinuum with nonvoid interior in cl $M(z)$. It follows that $H$ contains $x_{n}$ for arbitrarily large $n$. Let $h$ be a zero of $H$ and note that $[p, h] \cup H=X$. Since $[p, h] \cap H \subseteq E(h)$ it follows that $x \in H$ whenever $h<_{p} x$. Thus $H$ contains all the cluster points of the net $x_{n}$. Hence by the selection of $z, \operatorname{cl} M(z) \subseteq H$. From [9, Theorem 341, p. 139], we infer $\mathrm{cl} M(z)$ is indecomposable.

Now by the hypothesis, cl $M(z)$ is irreducible, or, equivalently, $\mathrm{cl} M(z)$ contains at least two composants. Let $K$ be a composant of $\mathrm{cl} M(z)$ which does not contain $z$.

To conclude the proof of the theorem we consider two cases: (a) $K \subseteq$ $[p, z]$, and (b) $K \nsubseteq[p, z]$.

Case (a). Recall that $K$ is dense in $\operatorname{cl} M(z)$. If $K \subseteq[p, z]$ then $\operatorname{cl} K=$ $\operatorname{cl} M(z) \subseteq[p, z]$, and hence $X=[p, z] \cup \operatorname{cl} M(z)=[p, z]$.

Case (b). Choose $x \in K-[p, z]$. Then since $X$ is a chain, $x \in M(z)$. Apply Claim 2 to the continuum $[p, x]$. We infer that $\operatorname{cl}(M(z) \cap[p, x])$ is a subcontinuum of $[p, x]$. Since $x, z \in \operatorname{cl}(M(z) \cap[p, x]) \subseteq \operatorname{cl} M(z)$ and $\operatorname{cl} M(z)$ is irreducible between $x$ and $z$ it follows that $\operatorname{cl} M(z)=\operatorname{cl}(M(z) \cap[p, x]) \subseteq[p, x]$. Hence, $X=[p, z] \cup \mathrm{cl} M(z) \subseteq[p, x] \subseteq X$; i.e., $X=[p, x]$. This concludes the proof of the theorem.

Corollary 1.3. Suppose $\Gamma_{p} \cup \Gamma_{p}^{-1}$ is a closed subset of $X \times X$. If $\left\{\left[p, x_{n}\right]\right\}$ is a nest of chains then $\operatorname{cl}\left(\cup_{n}\left[p, x_{n}\right]\right)$ is irreducible between $p$ and some other point.

2. Smooth continua. The continuum $Z$ is smooth at the point $q$ [8] provided $Z$ is hereditarily unicoherent at $q$ and for each net $z_{n} \in Z$ the condition $\lim _{n} z_{n}=z$ implies $\operatorname{Lim}_{n}\left[q, z_{n}\right]=[q, z]$. The continuum $Z$ is smooth if there exists a point $q$ such that $Z$ is smooth at $q$.

A generalized tree is an arcwise connected smooth continuum. A smooth dendroid is a metrizable generalized tree. According to Lemma 1.1 (vii) and [10] 
the above definition of generalized tree is equivalent to the definition originally given by Ward [20].

For the continuum $X$, which is hereditarily unicoherent at $p$, denote by $D(X, p)$ the set of all irreducible subcontinua of the form $[p, x]$. We view $D(X, p)$ as a subspace of $2^{X}$, the space of nonempty closed subsets of $X$ with the Vietoris topology [16]. Let $\eta_{p}: X \rightarrow O(X, p)$ denote the natural function defined by $\eta_{p}(x)=$ $[p, x]$. The space $\mathcal{D}(X, p)$ is studied in [14] when $X$ is a dendroid. Note that in this more general setting $\eta_{p}$ need not be one to one; indeed $\eta_{p}$ is one to one if and only if $\leqslant_{p}$ is a partial order.

The theorem below is proved in [14] for dendroids. The proof easily generalizes to nonmetric continua.

THEOREM 2.1. The continuum $X$ is smooth at $p$ if and only if the function $\eta_{p}: X \rightarrow O(X, p)$ is continuous.

For the continuum $X$, which is hereditarily unicoherent at $p$, we define an equivalence relation $\rho$ on $X$ by $(x, y) \in \rho$ if and only if $[p, x]=[p, y]$. Note that the equivalence class containing $x$ is $E(x)$. Let $\phi: X \rightarrow X / \rho$ denote the natural quotient map.

In case $X$ is smooth at $p$ Gordh [8] showed this relation defines an upper semicontinuous monotone decomposition of $X$ whose decomposition space is a generalized tree.

LEMMA 2.2. If $Y \subseteq X$ is a subcontinuum containing $p$ then $\phi^{-1}(\phi(Y))=Y$.

PROOF. We show only $\phi^{-1}(\phi(Y)) \subseteq Y$. If $x \in \phi^{-1}(\phi(Y))$ there exists $y \in$ $Y$ such that $\phi(x)=\phi(y)$. Since $Y$ is hereditarily unicoherent at $p, x \in[p, x]=$ $[p, y] \subseteq Y$.

3. Property $\alpha$ at $p$. The continuum $X$ has property $\alpha$ at $p$ if whenever $C$ is a subcontinuum containing $p$ and $C \subseteq[p, x]$ for some $x \in X$ it follows that $C=[p, c]$ for some $c \in C$. Observe that if $X$ is arcwise connected then $X$ has property $\alpha$ at $p$.

EXAmple 3.1. Let $P$ by the pseudo arc (see [9, p. 143]). Recall that $P$ is a metrizable hereditarily indecomposable continuum and is homeomorphic to each of its nondegenerate subcontinua. Using these facts it is not difficult to verify that $P$ is hereditarily unicoherent, $P$ contains no arcs, and $P$ has property $\alpha$ at each of its points.

THEOREM 3.2. If the continuum $X$ is smooth at $p$ then $X$ has property $\alpha$ at $p$.

Proof. By $[8$, Theorem 4.1(ii), p. 56] it follows that $\phi([p, x])=[\phi(p), \phi(x)]$ is an arc in the generalized tree $X / \rho$ for each $x \in X$. Let $C \subseteq X$ be a sub- 
continuum satisfying $p \in C \subseteq[p, x]$ for some $x \in X$. Then $\phi(p) \in \phi(C) \subseteq$ $[\phi(p), \phi(x)]$. Since $X / \rho$ has property $\alpha$ at $p$ there exists $c \in C$ such that $\phi(C)=$ $[\phi(p), \phi(c)]$. Finally, by Lemma $2.2, C=[p, c]$.

THEOREM 3.3. The continuum $X$ has property $\alpha$ at $p$ if and only if $[p, x]$ is a chain for each $x \in X$.

Proof. Assume $X$ has property $\alpha$ at $p$. For $y$ and $z$ in $[p, x]$ there exists $w \in[p, x]$ such that $[p, y] \cup[p, z]=[p, w]$. If $w \in[p, y]$ then $z \leqslant_{p} y$; if $w \in[p, z]$ then $y \leqslant_{p} z$. The converse follows immediately from Theorem 1.2.

COROLlaRY 3.4. If $X$ has property $\alpha$ at $p$ then $\leqslant_{p}$ is order dense (i.e., if $x<_{p} y$ there exists $z \in X$ such that $x<_{p} z<_{p} y$ ).

Proof. Suppose $x<_{p} y$ and $x<_{p} z<_{p} y$ for no $z \in X$. Then $[p, y]-$ $[p, x]$ is a nonempty open (in $[p, y]$ ) subset of $E(y)$ which contradicts Lemma 1.1(ii).

There exist continua for which $\leqslant_{p}$ is order dense but fail to have property $\alpha$ at $p$ (see Example 4.4).

4. Weakly smooth continua. The continuum $Z$ is weakly smooth at the point $q$ provided $Z$ is hereditarily unicoherent at $q, Z$ has property $\alpha$ at $q$, and the Li-condition:

$$
\begin{aligned}
& \text { for each convergent net } z_{n} \text { in } Z \text {, there exists } \\
& z \in Z \text { such that } \mathrm{Li}_{n}\left[q, z_{n}\right]=[q, z]
\end{aligned}
$$

holds. The continuum $Z$ is weakly smooth if there exists a point $q$ such that $Z$ is weakly smooth at $q$.

In case $Z$ is a dendroid this definition agrees with the definition of weakly smooth dendroids [14]. Also observe that in view of Theorem 3.2 a smooth continuum is weakly smooth.

THEOREM 4.1. If the continuum $X$ has property $\alpha$ at $p$ and is irreducible between $p$ and $q$ then $X$ is weakly smooth at $p$.

Proof. It suffices to show the $\mathrm{Li}$ condition holds. Let $x_{n}$ be a convergent net in $X$. By [8, Theorem 2.2, p. 53], $\mathrm{Li}_{n}\left[p, x_{n}\right]$ is a subcontinuum of $X$. Since $X=[p, q]$ and $X$ has property $\alpha$ at $p$ it follows that $\operatorname{Li}_{n}\left[p, x_{n}\right]=[p, x]$ for some $x \in X$. Thus, $X$ is weakly smooth at $p$.

Gordh $[8$, Theorem 3.3, p. 55] proved each smooth irreducible continuum is of type $A^{\prime}$. This is not the case for weakly smooth continua; Theorem 4.1 and the discussion in Example 3.3 imply the pseudo arc is weakly smooth.

Theorem 4.2. Let the continuum $X$ have property $\alpha$ at $p$. Then $X$ is 
weakly smooth at $p$ (i.e., the $\mathrm{Li}$ condition holds) if and only if $D(X, p)$ is a compact subset of $2^{X}$.

PRoof. Assume $X$ is weakly smooth at $p$. Since $2^{X}$ is a compact Hausdorff space $[16$, Theorem 4.9, p. 163] it suffices to show $D(X, p)$ is closed. Let $\left[p, x_{n}\right]$ be a net in $\mathcal{O}(X, p)$ converging to a point $A \in 2^{X}$. By [8, Theorem 2.2, p. 53], it follows that $A$ is a subcontinuum of $X$. From the net $x_{n}$ in $X$ select a convergent subnet $x_{m}$. By hypothesis, $\mathrm{Li}_{m}\left[p, x_{m}\right]=[p, x]$ for some $x \in X$. Since $X$ has property $\alpha$ at $p$ we infer from $p \in A=\operatorname{Lim}_{n}\left[p, x_{n}\right]=\operatorname{Li}_{n}\left[p, x_{n}\right] \subseteq$ $\mathrm{Li}_{m}\left[p, x_{m}\right]=[p, x]$ that $A \in D(X, p)$.

Conversely, assume $D(X, p)$ is compact and let $x_{n}$ be a convergent net in $X$. From the net $\left[p, x_{n}\right]$ in $D(X, p)$ select a convergent subnet $\left[p, x_{m}\right]$, say, $\operatorname{Lim}_{m}\left[p, x_{m}\right]=[p, x]$. Again from property $\alpha$ at $p$ and $p \in \mathrm{Li}_{n}\left[p, x_{n}\right] \subseteq$ $\operatorname{Li}_{m}\left[p, x_{m}\right]=\operatorname{Lim}_{m}\left[p, x_{m}\right]=[p, x]$ we infer $X$ is weakly smooth at $p$.

We now obtain various characterizations of weakly smooth continua. In most cases we assume $X$ is of type $A^{\prime}$ at $p$. We make use of the fact that irreducible continua of type $A^{\prime}$ admit a monotone map onto a (generalized) $\operatorname{arc} I$ (see [7]).

THEOREM 4.3. Suppose $X$ is of type $A^{\prime}$ at $p$. If $D(X, p)$ is compact or the Li condition holds then $X$ has property $\alpha$ at $p$.

Proof. Let $C$ be a subcontinuum of $X$ satisfying $p \in C \subseteq[p, x]$ for some $x \in X$. Let $\left\{\left[p, x_{n}\right]\right\}$ be a decreasing nest of subcontinua maximal with respect to $C \subseteq\left[p, x_{n}\right] \subseteq[p, x]$. It follows from either hypothesis that $\operatorname{Lim}_{n}\left[p, x_{n}\right]=$ $\bigcap_{n}\left[p, x_{n}\right]=[p, q]$ for some $q \in[p, x]$. Observe that $C \subseteq[p, q]$ and let $f:[p, q] \rightarrow I$ be the monotone map of [7]. If $f(C) \subsetneq I$ then there exist $s$ and $t$ in $I$ such that $f(C)=[0, t] \subsetneq[0, s] \subsetneq I$. Now for $q^{\prime} \in f^{-1}(s), C \subseteq\left[p, q^{\prime}\right] \subsetneq$ $[p, q]$ which contradicts the minimality of $[p, q]$ [7, Theorem 2.3, p. 649]. Thus, $f(C)=I$ and by [7, Theorem 2.5, p. 650], $C=[p, q]$.

The condition that $X$ is of type $A^{\prime}$ at $p$ is necessary as the example below shows.

Example 4.4. Let $P$ be the pseudo arc and let $p, a$, and $b$ be points which lie in distinct composants of $P$. Let $X$ be the continuum obtained by collapsing the sets $\{a\} \times\{0,1\}$ and $\{b\} \times\{0,1\}$ in $P \times\{0,1\}$. It is not difficult to see that $X$ is hereditarily unicoherent at $(p, 0)$ and $(p, 1)$, and $\leqslant_{(p, 0)}$ is order dense.

Let $x \neq a \quad(y \neq b)$ be a point in the same composant of $P$ as the point $a$ (b). Then in $X$ the points $(x, 1)$ and $(y, 1)$ are not related by $\leqslant_{(p, 0)}$. It follows from Theorem 3.3 that $X$ does not have have property $\alpha$ at $p$. Hence $X$ is not weakly smooth at $(p, 0)$.

It is, however, true that $D(X,(p, 0))$ is compact and the $\mathrm{Li}$ condition holds. The details are left to the reader. 
Recall that

$$
\Gamma_{p}=\left\{(x, y) \in X \times X \mid x \leqslant_{p} y\right\} \quad \text { and } \Gamma_{p}^{-1}=\left\{(x, y) \in X \times X \mid(y, x) \in \Gamma_{p}\right\}
$$

In [14] it was proved that a dendroid $X$ is weakly smooth at $p$ if and only if $\Gamma_{p} \cup \Gamma_{p}^{-1}$ is closed. The next theorem extends this result to continua.

THEOREM 4.5. If the continuum $X$ is weakly smooth at $p$ then $\Gamma_{p} \cup \Gamma_{p}^{-1}$ is a closed subset of the product space $X \times X$. The converse holds if $X$ is of type $A^{\prime}$ at $p$.

Proof. Assume $X$ is weakly smooth at $p$ and let $\left(x_{n}, y_{n}\right)$ be a net in $\Gamma_{p} \cup \Gamma_{p}^{-1}$ converging to $(x, y)$. Without loss of generality there exists a subnet $\left(x_{m}, y_{m}\right)$ such that $\left(x_{m}, y_{m}\right) \in \Gamma_{p}$ for each $m$. Since $X$ is weakly smooth at $p$, there exists $z \in X$ such that $\mathrm{Li}_{m}\left[p, y_{m}\right]=[p, z]$. Since $[p, z]$ is a chain containing $x$ and $y$ it follows that $(x, y) \in \Gamma_{p} \cup \Gamma_{p}^{-1}$.

For the converse, we first show $[p, q]$ is a chain for each $q \in X$. It will then follow from Theorem 3.3 that $X$ has property $\alpha$ at $p$. Let $f:[p, q] \rightarrow I$ be the monotone map of [7]. Choose $x$ and $y$ in $[p, q]$ and assume, without loss of generality, $f(x) \leqslant f(y)$. If $f(x) \leqslant f(y)$ then $[p, y]$ is a subcontinuum of $[p, q]$ which meets $f^{-1}(f(p))$ and $f^{-1}(f(y))$. Hence, by [7, Theorem 2.3, p. 649], $x \in f^{-1}(f(x)) \subseteq[p, y]$; i.e., $x \leqslant p$. Assume $f(x)=f(y)$ and $(x, y) \notin$ $\Gamma_{p} \cup \Gamma_{p}^{-1}$; then there exists an open neighborhood $U$ of $x$ satisfying $(x, y) \in U$ $\times\{y\} \subseteq X \times X-\left(\Gamma_{p} \cup \Gamma_{p}^{-1}\right)$. Now if $z \dot{\in} U$ and $f(z) \leqslant f(y)$ or $f(y) \leqslant f(z)$ it follows from the above argument that $(z, y) \in \Gamma_{p} \cup \Gamma_{p}^{-1}$. Thus, from the choice of $U$ we infer $U \subseteq f^{-1}(f(y))$. This contradicts [7, Theorem 2.7, p. 650]

To complete the proof we show the Li condition holds. Let $x_{n}$ be a convergent net in $X$. Since each $\left[p, x_{n}\right]$ is a chain and $\Gamma_{p} \cup \Gamma_{p}^{-1}$ is closed it follows that $\mathrm{Li}_{n}\left[p, x_{n}\right]$ is a chain. From Theorem 1.2 we infer $\mathrm{Li}_{n}\left[p, x_{n}\right]=[p, x]$ for some $x \in X$. Thus, $X$ is weakly smooth at $p$.

The next characterization of weakly smooth continua is in terms of the set function $T$ on $X$. For a continuum $Y$ and a point $y \in Y$ let $T(y)$ denote the set of all points $z \in Y$ such that each subcontinuum containing $z$ in its interior must also contain $y$. It is known [4] that $T(y)$ is a continuum.

Gordh [5] showed the continuum $X$ is smooth at $p$ if and only if $T(x) \subseteq$ $M(x)$ for each $x \in X$. We generalize this characterization to weakly smooth continua. The proof is a modification of an argument used by Charatonik and Eberhart [3, Theorem 5, p. 302].

THEOREM 4.6. If $X$ is weakly smooth at $p$ then $T(x) \subseteq L(x) \cup M(x)$ for each $x \in X$. The converse holds if $X$ is of type $A^{\prime}$ at $p$.

Proof. Suppose $X$ is weakly smooth at $p$ and for some $x \in X$ there exists $y \in X$ such that $y \in T(x)-(L(x) \cup M(x))$. Thus $(x, y) \notin \Gamma_{p} \cup \Gamma_{p}^{-1}$, and there 
exists an open neighborhood $V$ of $y$ such that $(x, y) \in\{x\} \times \operatorname{cl} V \subseteq X \times X-$ $\left(\Gamma_{p} \cup \Gamma_{p}^{-1}\right)$.

Now consider the set $L(\mathrm{cl} V) \cup M(\mathrm{cl} V)=Y$. Since $Y=\bigcup\{[p, x] \subseteq X \mid$ $[p, x] \cap(\mathrm{cl} V) \neq \square\}$ it is clearly connected. Let $z_{n}$ be a net in $Y$ converging to $x \in X$. For each $n$ choose $a_{n} \in \mathrm{cl} V$ such that $\left(z_{n}, a_{n}\right) \in \Gamma_{p} \cup \Gamma_{p}^{-1}$. Since cl $V$ $\subseteq X$ and $\Gamma_{p} \cup \Gamma_{p}^{-1} \subseteq X \times X$ are closed it follows that $z \in Y$. Hence $Y$ is also closed. But then, $Y$ is a subcontinuum of $X$ which contains $y$ in its interior and misses $x$. This is a contradiction.

Conversely, if $X$ is of type $A^{\prime}$ at $p$ it suffices to show $\Gamma_{p} \cup \Gamma_{p}^{-1}$ is closed. Select a point $(x, y) \notin \Gamma_{p} \cup \Gamma_{p}^{-1}$; then $x \notin T(y)$ and $y \notin T(x)$. Let $M$ and $N$ be subcontinua of $X$ satisfying: (i) $x \in$ int $M \subseteq M \subseteq X-\{y\}$, and (ii) $y \in$ int $N \subseteq$ $N \subseteq X-\{x\}$. Define $M^{\prime}=[p, x] \cup M\left(N^{\prime}=[p, y] \cup N\right)$ and observe that (i) and (ii) hold with $M$ and $N$ replaced by $M^{\prime}$ and $N^{\prime}$, respectively.

Let $V=\left(X-N^{\prime}\right) \times$ int $N^{\prime}$. Then $V \subseteq X \times X-\Gamma_{p}$; for if $(z, w) \in V \cap \Gamma_{p}$ then $z \in[p, w] \subseteq N^{\prime}$ which is a contradiction.

Dually, if $\bar{W}=$ int $M^{\prime} \times\left(X-M^{\prime}\right)$ then $W \subseteq X \times X-\Gamma_{p}^{-1}$. closed.

Since $(x, y) \in V \cap W \subseteq X \times X-\left(\Gamma_{p} \cup \Gamma_{p}^{-1}\right)$ it follows that $\Gamma_{p} \cup \Gamma_{p}^{-1}$ is

For continua of type $A^{\prime}$ at $p$ we have shown:

THEOREM 4.7. If $X$ is of type $A^{\prime}$ at $p$ then the following are equivalent: (i) $X$ is weakly smooth at $p$; (ii) the $\mathrm{Li}$ condition holds; (iii) $O(X, p)$ is compact; (iv) $\Gamma_{p} \cup \Gamma_{p}^{-1}$ is closed; and (v) $T(x) \subseteq L(x) \cup M(x)$ for each $x \in X$.

We conclude this section with two structure theorems for $D(X, p)$ which were proved in [14] for weakly smooth dendroids.

THEOREM 4.8. If $X$ is weakly smooth at $p$ then $D(X, p)$ is an arcwise connected continuum which admits a closed order dense partial order $\leqslant$ induced by inclusion. In particular, for each $x \in X, \eta_{p}([p, x])$ is an arc in $D(X, p)$ with noncut points $\eta_{p}(p)=[p, p]$ and $\eta_{p}(x)=[p, x]$.

Proof. It is well known that $2^{X}$ admits a closed partial order $\leqslant$ defined by $A \leqslant B$ if and only if $A \subseteq B$. Clearly, $D(X, p)$ inherits this closed partial order. Note that $[p, a] \leqslant[p, b]$ if and only if $a \leqslant p$. Using Theorem 3.4 it is trivial to show $\leqslant$ is order dense and $\eta_{p}([p, x])$ is a closed chain (with respect to $\$$ ) for each $x \in X$. It follows from [10, Lemma 1, p. 922], that $\eta_{p}([p, x])$ is an arc with noncut points $[p, p]$ and $[p, x]$.

Corollary 4.9. If $X$ is smooth at $p$ then $\eta_{p}: X \rightarrow \mathcal{D}(X, p)$ is a monotone surjective map. Consequently, $\mathcal{O} X, p)$ is a generalized tree which is smooth at $p$. 
Proof. According to [8, Theorem 3.3, p. 53] and Theorem 1.1(iv), $\eta_{p}$ is monotone. By $[8$, Theorem 4.1, p. 56], $D(X, p)$ is hereditarily unicoherent at $\eta_{p}(p)$. Since $D(X, p)$ is arcwise connected, it is hereditarily unicoherent. The result now follows from Theorem 4.8.

At this point we pose a question: If $X$ is weakly smooth at $p$, is $D(X, p)$ a generalized tree which is smooth at $\eta_{p}(p)$ ? By Theorem 4.8 it is equivalent to ask if $\mathcal{D}(X, p)$ is hereditarily unicoherent at $\eta_{p}(p)$.

5. Images of weakly smooth continua. In this section we investigate various types of maps on weakly smooth continua and the extent to which they preserve weak smoothness. Compare these results with those of Gordh [8] and Charatonik and Eberhart [3].

Recall $X$ is a continuum which is hereditarily unicoherent at $p$.

LEMMA 5.1. Let $f: X \rightarrow Y$ be a map from $X$ into the compact Hausdorff space $Y$. Then the function $f^{\prime}: D(X, p) \rightarrow 2^{Y}$ defined by $f^{\prime}([p, x])=f([p, x])$ is continuous.

Proof. The proof follows immediately from [16, Theorem 5.10, p. 170].

Let $f: X \rightarrow Y$ be a map from $X$ into a continuum $Y$. The map is $\leqslant_{p}$-preserving if $Y$ is hereditarily unicoherent at $f(p)$ and $x \leqslant_{p} y$ implies $f(x) \leqslant_{f(p)} f(y)$.

THEOREM 5.2. Let $f: X \rightarrow Y$ be $a \leqslant p$-preserving map from $X$ onto $Y$. Then:

(i) $f([p, x])=[f(p), f(x)]$ for each $x \in X$;

(ii) if $X$ has property $\alpha$ at $p$ then $Y$ has property $\alpha$ at $f(p)$;

(iii) if $X$ is weakly smooth at $p$ then $Y$ is weakly smooth at $f(p)$; and

(iv) if $X$ is smooth at $p$ then $Y$ is smooth at $f(p)$.

Proof. (i) The argument of [3, Proposition 4, p. 309], holds for (nonmetric) continua.

(ii) Let $C$ be a subcontinuum of $Y$ satisfying $f(p) \in C \subseteq[f(p), f(x)]$ from some $x \in X$. By [3, Proposition 4, p. 309], the restriction of $f$ to $[p, x]$ is monotone; hence $f^{-1}(C) \cap[p, x]$ is a subcontinuum of $[p, x]$. Since $X$ has property $\alpha$ at $p$ there exists $c \in X$ such that $f^{-1}(C) \cap[p, x]=[p, c]$. It follows that $[f(p), f(c)]=f([p, c])=f\left(f^{-1}(C) \cap[p, x]\right)=C$; that is, $Y$ has property $\alpha$ at $f(p)$.

(iii) Since $Y$ has property $\alpha$ at $f(p)$, it suffices by (ii) to show $D(Y, f(p))$ is compact (Theorem 4.2). It follows from (i) that $f^{\prime}$ maps the compact $D(X, p)$ onto $D(Y, f(p))$.

(iv) Consider the diagram 


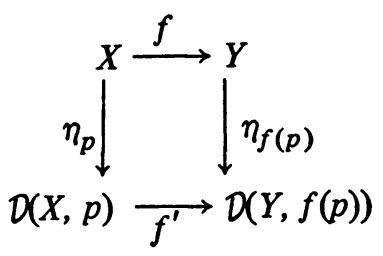

which is easily shown to commute; i.e., $f^{\prime} \eta_{p}=\eta_{f(p)} f$. The continuity of $f, f^{\prime}$, and $\eta_{p}$ assure the continuity of $\eta_{f(p)}$. By Theorem $2.1 Y$ is smooth at $f(p)$.

Corollary 5.3. Let $f: X \rightarrow Y$ be a monotone map from $X$ onto a compact Hausdorff space Y. Then (i)-(iv) of Theorem 5.2 hold.

Proof. The space $Y$ is a continuum which is hereditarily unicoherent at $p$ by $\left[8\right.$, Theorem 4.1, p. 56]. The map $f$ is $\leqslant_{p}$-preserving by $[3$, Proposition 4 , p. 309].

Gordh [8] originally proved the monotone image of a smooth continuum is smooth but his proof is different from the one presented here.

The full strength of $\leqslant_{p}$-preserving maps is not necessary to preserve weak smoothness of continua. A map $f: X \rightarrow Y$ from $X$ onto a continuum $Y$ which is hereditarily unicoherent at $f(p)$ is weakly $\leqslant_{p}$-preserving in case $x \leqslant_{p} y$ implies $f(x) \leqslant_{f(p)} f(y)$ or $f(y) \leqslant_{f(p)} f(x)$.

THEOREM 5.4. Let $f: X \rightarrow Y$ be a weakly $\leqslant_{p}$-preserving map from $X$ onto $Y$. Assume $Y$ is hereditarily unicoherent at $f(p)$ and is of type $A^{\prime}$ at $f(p)$. If $X$ is weakly smooth at $p$ then $Y$ is weakly smooth at $f(p)$.

Proof. It suffices to show $D(Y, f(p))$ is compact (Theorem 4.7). To this end we will show $f^{\prime}: D(X, p) \rightarrow 2^{Y}$ maps $D(X, p)$ onto $D(Y, f(p))$.

If $[p, x] \in D(X, p)$ then $[p, x]$ is a chain (with respect to $\leqslant_{p}$ ) and hence $f([p, x])$ is a chain (with respect to $\left.\leqslant_{f(p)}\right)$. By Theorem 1.2 there exists $y \in Y$ such that $f([p, x])=\bigcup\{[f(p), f(z)] \mid z \in[p, x]\}=[f(p), y] \in D(Y, f(p))$. Thus, $f^{\prime}$ maps $D(X, p)$ into $D(Y, f(p))$.

For any point $[f(p), f(x)] \in \mathcal{O}(Y, f(p))$ consider the closed set $A=[p, x]$ $\cap f^{-1}(f(x)) \subseteq[p, x]$. Let $z \in \min Z(\neq \square$ by Lemma 1.1(v)) and observe that $z$ is a zero of $Z$ since $Z$ is a chain. It follows that $[f(p), f(x)] \subseteq f([p, z]) \in$ $D(Y, f(p))$. If the inclusion is proper there exists a point $w<_{p} z$ such that $f(w)$ $=f(x)$, but this contradicts the definition of $z$. Hence $f^{\prime}$ is onto.

The weakly $\leqslant_{p}$-preserving image of a smooth continuum need not be smooth, even if both spaces are dendroids.

EXAMPLE 5.5. In the plane with polar coordinates join the point $p=$ $(0,0)$ with $a_{0}=(1,0)$ and with points $a_{n}=\left(1,1 / 2^{n-1}\right)$ for $n=1,2, \ldots$ by straight line segments. The dendroid $X$ obtained in this way is a harmonic fan 
with top $p$. A harmonic hooked fan $Y$ is obtained by joining the points $a_{n}$ in $X$ with $b_{n}=\left(1 / 2,3 / 4 \cdot\left(1 / 2^{n-1}\right)\right)$ by straight line segments.

Let $f(p)=p, f\left(a_{0}\right)=(1 / 2,0), f((1 / 2,0))=a_{0}$, and $f\left(a_{n}\right)=b_{n}$ for each $n=$ $1,2, \ldots$ Clearly $f$ can be extended to a weakly $\leqslant_{p}$-preserving map from $X$ onto $Y$. However, $X$ is smooth at $p$ while $Y$ is not smooth.

6. The spaces $X / \rho$ and $D(X / \rho, \phi(p))$. Recall the definition of the quotient space, $X / \rho$ (see $\S 2$ ). Of course $X / \rho$ is always connected but, in general, it may not be Hausdorff (e.g., let $X$ be the pseudo arc).

THEOREM 6.1. If $X / \rho$ is Hausdorff then the quotient map $\phi: X \rightarrow X / \rho$ is monotone, $X / \rho$ is a continuum which is hereditarily unicoherent at $\phi(p)$, and $\phi$ induces a homeomorphism $\phi^{\prime}$ from $D(X, p)$ onto $D(X / \rho, \phi(p))$.

Proof. If $X / \rho$ is Hausdorff then each point $\phi(x) \in X / \rho$ is closed. Hence $E(x)=\phi^{-1}(\phi(x)) \subseteq X$ is closed. By Theorem 1.12(i) each $E(x)$ is connected; thus $\phi$ is monotone. By [8, Theorem 4.1, p. 56], $X / \rho$ is hereditarily unicoherent at $\phi(p)$; so $D(X / \rho, \phi(p))$ is well defined. From Lemma 5.1, Corollary 5.3, and Lemma 2.2 we infer $\phi([p, x])=[\phi(p), \phi(x)]$ defines a one to one map from $\mathcal{D} X, p)$ onto $\mathcal{D}(X / \rho, \phi(p))$. It remains only to show the inverse function is continuous. (Since $X$ is not assumed to be weakly smooth at $p$ it does not follow that $O(X, p)$ is compact.)

Let $\phi\left(\left[p, x_{n}\right]\right)$ be a net in $D(X / \rho, \phi(p))$ converging to $\phi([p, x])$. We show $\operatorname{Lim}_{n}\left[p, x_{n}\right]=[p, x]$. Let $\left[p, x_{m}\right]$ be a convergent subnet of the net $\left[p, x_{n}\right]$ (one exists since $2^{X}$ is compact Hausdorff [16]).

We first show $[p, x] \subseteq \operatorname{Lim}_{m}\left[p, x_{m}\right]$. If $z \in[p, x]$ then $\phi(z) \in \phi([p, x])$ $=\operatorname{Lim}_{m} \phi\left(\left[p, x_{m}\right]\right)$ and there exist points $z_{m} \in\left[p, x_{m}\right]$ such that $\lim _{m} \phi\left(z_{m}\right)=$ $\phi(z)$. Let $z_{k}$ be a convergent subnet of $z_{m}$, say $\lim _{k} z_{k}=z^{\prime}$. By the continuity of $\phi, \lim _{k} \phi\left(z_{k}\right)=\phi\left(z^{\prime}\right)=\phi(z)$. It follows that $z \in[p, z]=\left[p, z^{\prime}\right] \subseteq$ $\operatorname{Ls}_{m}\left[p, x_{m}\right]=\operatorname{Lim}_{m}\left[p, x_{m}\right]$.

We now show the reverse inclusion. If $z \in \operatorname{Lim}_{m}\left[p, x_{m}\right]$ there exist points $z_{m} \in\left[p, x_{m}\right]$ such that $\lim _{m} z_{m}=z$. Since $\phi$ is continuous, $\lim _{m} \phi\left(z_{m}\right)=$ $\phi(z)$. It follows that $\phi(z) \in \mathrm{Ls}_{m} \phi\left(\left[p, x_{m}\right]\right)=\operatorname{Lim}_{m} \phi\left(\left[p, x_{m}\right]\right)=\phi([p, x])$. Hence $z \in[p, x]$ by Lemma 2.2.

Thus, $[p, x]$ is the only cluster point of the net $\left[p, x_{n}\right]$. Since $2^{X}$ is compact Hausdorff it follows that $\operatorname{Lim}_{n}\left[p, x_{n}\right]=[p, x]$.

This completes the proof of the theorem.

Corollary 6.2. Suppose $X / \rho$ is Hausdorff. If $X$ is weakly smooth at $p$ then $X / \rho$ is weakly smooth at $p$. The converse is true if $X$ is of type $A^{\prime}$ at $p$.

Proof. The first statement follows from Corollary 5.3; the converse from Theorem 4.7. 
We now prove the converse of Gordh's decomposition for smooth continua [9, Theorem 5.3, p. 57]. A proof due to T. Mackowiak [15, Theorem 3.1, p. 177] easily generalizes to (nonmetric) continua but we present an alternate proof here.

THEOREM 6.3. If $X / \rho$ is a continuum which is smooth at $\phi(p)$ then $X$ is smooth at $p$.

Proof. Since $\phi$ is monotone the diagram below commutes.

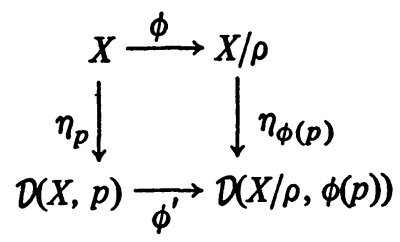

Since $\phi$ is continuous, $\eta_{\phi(p)}$ is continuous (Theorem 2.1), and $\phi^{\prime}$ is a homeomorphism (Theorem 6.1) we infer $\eta_{p}$ is continuous. Thus $X$ is smooth at $p$.

7. A quasi order characterization. Ward [20] characterized generalized trees as compact Hausdorff spaces which admit a partial order satisfying certain properties. In [13] an analogous quasi order characterization of smooth continua was obtained. In this section we establish a quasi order characterization of weakly smooth continua.

THEOREM 7.1. Let $Z$ be a compact Hausdorff space such that each of its indecomposable subcontinua is irreducible. Then $Z$ is a continuum which is weakly smooth at $p$ if and only if $Z$ admits a quasi order $\Gamma$ satisfying:

(i) $\Gamma \cup \Gamma^{-1}$ is closed;

(ii) there exists $p \in \cap\{L(x) \mid x \in Z\}$ and each $L(x)$ is a connected chain; and

(iii) if $Y$ is a closed connected subset of $Z$ with $p \in Y$, then $L(y) \subseteq Y$ for each $y \in Y$.

Proof. If $Z$ is weakly smooth at $p$ then the weak cut point order with respect to $p$ satisfies (i)-(iii). To prove the converse, we show the quasi order $\Gamma_{p}$ defined by $(x, y) \in \Gamma_{p}$ if and only if $x \in \operatorname{cl} L(y)$ is the weak cut point order with respect to $p$.

For notational convenience, let $L_{p}(x)=\operatorname{cl} L(x)$ for each $x \in Z$ and if $Y \subseteq$ $Z$ is a chain with respect to $\Gamma\left(\Gamma_{p}\right)$ we will write " $Y$ is a $\Gamma\left(\Gamma_{p}\right)$-chain".

Observe that $L_{p}(x)$ is a $\Gamma$-chain (and hence a $\Gamma_{p}$-chain) for each $x \in X$ since: $L(x) \times L(x) \subseteq \Gamma \cup \Gamma^{-1}$ by (ii); and thus by (i),

$$
L(x) \times L(x) \subseteq L_{p}(x) \times L_{p}(x) \subseteq \Gamma \cup \Gamma^{-1} \subseteq \Gamma_{p} \cup \Gamma_{p}^{-1}
$$


The above inclusions also show $\Gamma_{p} \cup \Gamma_{p}^{-1}=\Gamma \cup \Gamma^{-1}$. For if $(x, y) \in$ $\Gamma_{p} \cup \Gamma_{p}^{-1}$ then $(x, y) \in L_{p}(y) \times L_{p}(y) \subseteq \Gamma \cup \Gamma^{-1}$.

Consequently, the quasi order $\Gamma_{p}$ not only satisfies properties (i)-(iii) with $\Gamma$ replaced by $\Gamma_{p}$ and $L(x)$ replaced by $L_{p}(x)$ but also the property that each $L_{p}(x)$ is a continuum.

Using (ii) and (iii) it is straightforward to verify that $Z$ is a continuum which is hereditarily unicoherent at $p ; \Gamma_{p}$ is the weak cut point order with respect to $p$; and each $L_{p}(x)=[p, x]$ is a $\Gamma_{p}$-chain. Hence by Theorem $3.3, Z$ has property $\alpha$ at $p$.

We conclude the proof by showing the Li condition holds. Let $x_{n}$ be a convergent net in $Z$. Since $\Gamma_{p} \cup \Gamma_{p}^{-1}$ is closed it follows that the continuum $\mathrm{Li}_{n}\left[p, x_{n}\right]$ is a $\Gamma_{p}$-chain. Thus by Theorem $1.2, \mathrm{Li}_{n}\left[p, x_{n}\right]$ is of the form $[p, x]$ for some $x \in Z$.

We remark that the quasi order $\Gamma$ may be properly contained in $\Gamma_{p}$. In fact, one can define a linear partial order $\Gamma$ on the closure of the "sin $(1 / x)$-curve" satisfying (i)-(iii) with $p=(1, \sin 1)$.

8. Arboroids. Recall an arboroid is a hereditarily unicoherent arcwise connected continuum. The first two theorems of this section were proved in [14] for dendroids. Their proofs generalize in a straightforward manner.

THEOREM 8.1. Let $X$ be an arboroid. If $Y \subseteq X$ is a subcontinuum and $q$ is the zero of $Y$ with respect to $\leqslant_{p}$ then $Y$ is weakly smooth at $q$ and $\eta_{p}(Y) \subseteq$ $D(X, p)$ is homeomorphic to $D(Y, q)$.

THEOREM 8.2. The arboroid $X$ is a generalized tree if and only if there exists a homeomorphism $h: X \rightarrow D(X, p)$ from $X$ onto $D(X, p)$ for some $p \in X$.

We remark that Theorem 8.1 is not valid for arbitrary weakly smooth (or smooth) continua since they may contain nonunicoherent subcontinua. Also, in Theorem 8.2 the arboroid $X$ need not be smooth at $p$ (see [14, Example 2, p. 116]).

Since an arboroid is hereditarily decomposable [1] it is of type $A^{\prime}$ at $p$. Thus, Theorem 4.7 is valid for arboroids.

Finally, a slight modification of conditions (i)-(iii) of Theorem 7.1 yields a characterization of weakly smooth arboroids.

THEOREM 8.3. Let $Z$ be a compact Hausdorff space such that each of its indecomposable subcontinua is irreducible. A necessary and sufficient condition that $Z$ be a weakly smooth arboroid is that $Z$ admit a partial order $\Gamma$ satisfying (i) and (iii) of Theorem 7.1 and (ii') there exists $p \in \bigcap\{L(x) \mid x \in Z\}$ and if $(x, y) \in \Gamma$ then $M(x) \cap L(y)$ is a closed connected chain. 
Proof. Since $M(p)=Z$, (ii') implies (ii). Thus $Z$ is a continuum which is weakly smooth at $p$. Since each $L(x)$ is closed it follows that $\Gamma=\Gamma_{p}$. According to Corollary 3.4 and [18, Lemma 1, p. 922], $Z$ is arcwise connected. Consequently, $Z$ is an arboroid ( $Z$ is hereditarily unicoherent by Lemma 1.1(viii)).

\section{REFERENCES}

1. D. P. Bellamy, Composants of Hausdorff indecomposable continua; a mapping approach, Pacific J. Math. 47 (1973), 303-309. MR 48 \#9679.

2. J. J. Charatonik, On ramification points in the classical sense, Fund. Math. 51 (1962/63), 229-252. MR 26 \#743.

3. J. J. Charatonik and C. A. Eberhart, On smooth dendroids, Fund. Math. 67 (1970), 297-322. MR 43 \#1129.

4. H. S. Davis, D. P. Stadtlander and P. M. Swingle, Properties of the set function $T^{n}$, Portugal. Math. 21 (1962), 113-133. MR 25 \#5501.

5. G. R. Gordh, Jr., Concerning closed quasi-orders on hereditarily unicoherent continua, Fund. Math. 78 (1973), no. 1, 61-73. MR 48 \#1196.

6. Monotone decompositions of irreducible Hausdorff continua, University of California at Riverside, Ph. D. Dissertation, 1971.

7. Monotone decompositions of irreducible Hausdorff continua, Pacific J. Math. 36 (1971), 647-658. MR 43 \#6882.

8. - On decompositions of smooth continua, Fund. Math. 75 (1972), 51-60. MR 47 \#5846.

9. J. G. Hocking and G. S. Young, Topology, Addison-Wesley, Reading, Mass., 1961. MR 23 \#A2857.

10. R. J. Koch and I. S. Krule, Weak cutpoint ordering on hereditarily unicoherent continua, Proc. Amer. Math. Soc. 11 (1960), 679-681. MR 22 \#11356.

11. K. Kuratowski, Topology. Vol. I., 5th ed., PWN, Warsaw; Academic Press, New York, 1968. MR 36 \#840.

12. Topology. Vol. II, 4th ed., PWN, Warsaw; Academic Press, New York, 1968. MR 41 \#467. (1974).

13. L. Lum, A quasi order characterization of smooth continua, Pacific J. Math. 53 \#12483.

14. Weakly smooth dendroids, Fund. Math. 83 (9174), 111-120. MR 48

15. T. Maćkowiak, Some characterizations of smooth continua, Fund. Math. 79 (1973), 173-186. MR 47 \#9566.

16. E. A. Michiael, Topologies on spaces of subsets, Trans. Amer. Math. Soc. 71 (1951), 152-182. MR 13, 54.

17. E. S. Thomas, Jr., Monotone decompositions of irreducible continua, Rozprawy Mat. 50 (1966). MR 33 \#907.

18. L. E. Ward, Jr., A fixed point theorem for multi-valued functions, Pacific J. Math. 8 (1958), 921-927. MR 21 \#2215.

19. $\longrightarrow$ A note on dendrites and trees, Proc. Amer. Math. Soc. 5 (1954), 992-

994. MR 17, 180.

20. - Mobs, trees, and fixed points, Proc. Amer. Math. Soc. 8 (1957), 798-

804. MR $20 \# 3516$.

21. Partially ordered topological spaces, Proc. Amer. Math. Soc. 5 (1954), 144-161. MR 16, 59.

DEPARTMENT OF MATHEMATICS, UNIVERSITY OF TENNESSEE, KNOXVILLE, TENNESSEE 37916

Current address: Department of Mathematics, Salem College, Winston-Salem, North Carolina 27108 